\title{
Workshop Report-Challenges in Dissolution Testing: Equivalence and Surrogates
}

\author{
Professor Izzy Kanfer \\ Rhodes University, Grahamstown, South Africa
}

A Dissolution Workshop, co-sponsored by AAPS and Rhodes University, was held at Rhodes University, Grahamstown, South Africa on 9-10 December 2009. The workshop was part of the outreach effort of the AAPS In Vitro Release and Dissolution Testing Focus Group. Members of the organizing committee were Professor Izzy Kanfer - Chair, Ms. Vivian Gray - Co-Chair, Mr. Michael Aeroboe, Dr. Srinivas Patnala, Dr. Michael F. Skinner, and Professor R. B. Walker.

\section{DAY 1}

\section{CONCEPTS AND APPLICATIONS OF DISSOLUTION TESTING}

\section{SESSION 1}

The Workshop was officially opened by the Chairperson, Professor Izzy Kanfer, Emeritus Professor, Faculty of Pharmacy, Rhodes University.

The first speaker was Dr. Gordon Amidon of the College of Pharmacy, University of Michigan, Ann Arbor.

Title of Talk: THE BIOPHARMACEUTICS CLASSIFICATION SYSTEM (BCS): FROM THEORY TO APPLICATION TO PRODUCT DEVELOPMENT.

Dr. Amidon underlined that "Bioequivalence" (BE) connects the product in the bottle with the claims on the label! The FDA adopted the premise that similar drug plasma levels following the administration of immediaterelease solid oral dosage forms will provide similar pharmacodynamics and hence serve as a measure to determine bioavailability and to establish bioequivalence between products. However, in August 2000 the FDA issued a Guidance for Industry (BCS Guidance) entitled Waiver of in vitro Bioavailability and Bioequivalence Studies for Immediate-Release Solid Oral Dosage Forms Based on a Biopharmaceutics Classification System. This Guidance extended the principles for the evaluation of $B E$ on the basis that similar in vivo dissolution should provide similar plasma levels and link in vitro dissolution to in vivo dissolution.

The BCS considers that drug is absorbed through the intestinal membrane at a rate that is proportional to the concentration at the membrane surface. The dissolution of the drug product in vivo determines the membrane surface area concentration of drug and hence similar in vivo dissolution should result in similar in vivo absorption and consequently similar systemic availability (BE).
Dr. Amidon contended that $\mathrm{BE}$ is a question of dissolution and absorption, and hence BE in vitro methodology must be able to capture the most important rate-controlling process, in vivo dissolution.

Dr. Amidon described the mechanistic approach taken by the BCS to setting bioequivalence standards by considering mass transport in the Gl tract. He also described the various classes and the conditions required for classification according to the BCS and the associated dissolution testing requirements. He compared the current European Agency for the Evaluation of Medicinal Products (EMA), Committee for Proprietary Medicinal Products (CPMP) Note for Guidance on the Investigation of Bioavailability and Bioequivalence with the FDA BCS Guidance, which is quite similar. He also discussed the WHO Expert Committee document on Specifications for Pharmaceutical Preparations, which describes the recommendations for $\mathrm{BE}$.

Dr. Amidon concluded that the BCS provides a new paradigm to reduce unnecessary in vivo studies and has utility to increase product quality. Since it is based on scientific principles, it may be extendable to other situations that may influence BE such as food effects. He emphasized that the BCS Guidance was intended to provide information for a waiver of in vivo $B E$ studies and not as a waiver of BE per se.

The next speaker, Vivian Gray of V.A. Gray Consulting, presented a talk entitled:DETERMINING SIMILARITY OF PRODUCTS $-f_{2}$ CRITERION AND VARIABILTY OF DISSOLUTION TESTS.

Vivian discussed dissolution profiles as the basis for granting biowaivers for new drug and generic drug applications and for different strengths, and for assuring product sameness under FDA, WHO, Japanese, and European guidances. She described the use of the $f_{1}$ and $f_{2}$ factors as bioequivalence tools. She referred to several FDA Guidances. She discussed the different wording from $\mathrm{WHO}, \mathrm{FDA}$, and EMEA regarding the appropriate dissolution result measurement after $85 \%$ dissolution. The improper application of the criteria and explanations of failures were discussed using several examples. The Pharmaceutical and Medical Devices Agency (PMDA) of Japan does not recognize the BCS for a biowaiver for any drug class although their guidelines combine the BCS waiver concept with SUPAC (scale up and post approval changes) and include modified-release dosage forms. 
Vivian further discussed the $\mathrm{WHO}$ requirements where extensions of biowaivers have been recommended for (1) BCS Class 3 if $85 \%$ is dissolved in 15 min using the three media and (2) BCS Class 2 weak acids if the API has a dose solubility ratio of $250 \mathrm{~mL}$ or less at $\mathrm{pH} 6.8$ and the multisource product is rapidly dissolving (no less that $85 \%$ in $\mathrm{pH} 6.8$ in $30 \mathrm{~min}$ ) and has a similar $f_{2}$ at all $\mathrm{pH}$ values. A paddle speed of $75 \mathrm{rpm}$ is allowed. Various examples were described to account for failures to meet $f_{2}$ requirements. These include factors such as product age (especially with capsules), prudent selection of innovator lots, solubility differences due to particle size, effect of excipient matrix, and disintegration properties. Minimizing variability was emphasized as an important aspect of the method as well as the selection of test conditions. In addition, earlier time points $(5,10 \mathrm{~min}$ ) may need to be added to obtain a good profile. Hydrodynamic aspects should be observed, and artifacts such as sticking, coning, clogging, and floating should be minimized.

\section{SESSION 2}

Starting session 2 was Terry Way, Senior International Representative for USP in Europe, Middle East, and Africa, and the title of his talk was: ENSURING COMPARABILITY IN DISSOLUTION TESTING.

Terry began by describing the USP Organization, a private, nonprofit organization established in 1820, which is recognized in more than 130 countries with locations in Rockville, MD, USA; Basel, Switzerland; Hyderabad, India; Shanghai, China; and São Paulo, Brazil.

He emphasized that dissolution assesses the performance of products, and to be effective, the test should be predictive, comparative, discriminatory, and reproducible. Furthermore, he asserted that dissolution testing is a sequence of complex processes. He stated that sources of variability might arise from the dissolution testing apparatus that generates the results including its environment, the details of the testing procedure, the analytical method used to quantify the dissolved drug substance in the test solution, the samples themselves and their intrinsic variability, and finally the analyst.

He discussed some issues surrounding the dissolution vessels. Because dissolution vessels are usually made by traditional glass-blowing techniques and each vessel is more or less unique since they are effectively hand-made, they may have individual flaws (the hemisphere may be distorted due to the method of manufacture) that add to hydrodynamic variability. He stated that even with perfect manufacture,"within specification" differences are significant. Furthermore, he emphasized that where possible, apparatus of closely similar dimensions and performance should be used for comparisons of product performance. He referred to the current harmonized USP General Chapter $<711>$ Dissolution, which allows for wider tolerances, encompassing the variety of apparatuses used worldwide that should be considered acceptable for meaningful comparisons. He also described the USP Dissolution Toolkit involving a Dissolution Procedure for the Mechanical Calibration and Performance Verification Test (www.usp.org/pdf/EN/dissolutionProcedureToolkit200710-04.pdf). It states "Assurance of the integrity of the dissolution procedure is achieved through careful assembly qualification, analyst training, and use of validated analytical procedures."The Toolkit provides enhanced procedures and control limits for mechanical qualification and a guide to producing better results from the Performance Verification Test. Terry stated that mechanical calibration alone is not sufficient to ensure consistency and comparability of measurements obtained with a dissolution test system and that the USP Performance Verification Test (PVT) is a holistic approach to verify dissolution apparatus operation. A periodic PVT together with careful mechanical calibration is important to ensure consistent results, and Prednisone RS Tablets are suitable for the PVT of USP Apparatus 1 and 2, whereas the test using Salicylic Acid RS Tablets will not be required for the PVT of USP Apparatus1 and 2 starting Dec. 1, 2009.

The next speaker was Dr. Johannes Krämer (PHAST, Germany), whose presentation was entitled:

\section{ACHIEVING OPTIMUM LEVELS of DISCRIMINATION in DISSOLUTION TESTING ... BUT HOW?}

Dr. Krämer stated that one of the most important goals of dissolution testing is the prediction of the clinical performance of drugs and that a great deal of emphasis is being placed on "Biorelevant" dissolution testing. He described the main goal as being the use of dissolution testing to evaluate the in vitro performance of drug products and in early development, to model in vitro findings with the goal of identifying the best delivery concept. In later phase development, the goals of dissolution testing include its application to predict differences in bioperformance within product lifecycle and to ascertain constant quality within a meaningful range. In addition, it was considered optimal to evaluate the impact of critical quality parameters on the in vitro performance of drugs. However, a prerequisite was to use standardized dissolution equipment and "reagents" for precise testing to measure quality differences and to link to relevant in vitro parameters.

Dr. Krämer described the concept of "Mapping," which involves a consideration of critical manufacturing variables, critical formulation variables, and critical processing variables. The "variables" include those materials and methods used in the manufacturing process that can significantly affect drug release from the product. He emphasized the importance of the need to develop product specifications with bioavailability implications. The issue of in vitro-in vitro (IVIVC) relationships was discussed and the use of the TNO TIM-1 model to simulate the full Gl tract was described. 
As far as the predictive power of dissolution testing is concerned, in practice, a rank order may be established. With regard to the bioperformance of drugs, Dr. Krämer is of the opinion that the BCS is a relevant starting point. Furthermore, he asserted that correlation of in vitro and in vivo data is possible citing the USP from 1975, which states that "...in many cases it is possible to correlate dissolution rates with biological availability of the active ingredient."

The BCS predictability was greater for immediaterelease dosage forms than for modified-release, and the use of dissolution media such as fasted-state simulated in testinal fluid (FaSSIF) and fed-state simulated intestinal fluid (FeSSIF), and others is of greater significance for immediate-release products. Dr. Krämer concluded his presentation by emphasizing that the geometry of dosage forms, the pharmacokinetic characteristics of the drug substance, and dosage form properties require consideration of Gl physiology. In addition, the discriminative power has to strike a balance between patient and manufacturer risk on a qualitative basis as well as a quantitative approach.

\section{PANEL DISCUSSION}

The first question of the session was how does the BE $90 \% \mathrm{Cl}$ of $80-125 \%$ relate to dissolution requirements for $\mathrm{BE}$ ?

The panel described $80-125 \%$ as the FDA "Gold Standard" for in vivo BE. It was explained that when the conditions for a BE waiver of BCS 1 drugs were proposed, they were based on simulations where it could be shown that even if the dissolution rate was theoretically slowed to one hour for a BCS Class 1 drug, that product could still meet the $80-125 \%$ requirement. However, for a BCS 3 drug, a dissolution specification of $60 \mathrm{~min}$ would not be good enough. Describing the actual choice of acceptance "numbers," Dr. Amidon stated that the actual quantitative numbers can be considered somewhat arbitrary and that the choice of numbers for the guidance was deliberatively conservative. When asked whether the numbers could be relaxed, he explained that the major Pharma companies wanted high standards, but he felt that the standards were too high and could be relaxed. He commented that over the past 10 years or more there had been proposals made at several conferences and workshops to revisit and relax the BCS standards but to-date, nothing has happened.

The next question and discussion related to narrow therapeutic index (NTI) drugs and whether stricter standards should be applied to that class of drug products. The panel response was that as long as the performance standards of the innovator product are met, there should be no need to impose further constraints on a generic product. This concern is apparently the result of an erroneous perception that medical doctors and practitioners feel that there is a need to protect the patient using $\mathrm{NTI}$ drugs. The issue of different dosage forms was then brought up as an analogous example where in the USA, tablets may not be substituted for capsules (i.e., the dosage form must be pharmaceutically equivalent and bioequivalent for substitution). This requirement is neither rational nor scientific but is presumably based on the possibility of causing patient confusion. If a patient is given a tablet and then, on another occasion, a capsule, and the pharmacist says it is the same, will the patient think they are the same? Probably not, hence noninterchangeability between different dosages forms can be justified on the basis that it avoids confusion. Professor Kanfer concurred that there was no scientific basis to have stricter standards for NTIs.

The issue regarding variations between results obtained using different dissolution equipment was then raised. One of the participants indicated her concern that she had experienced differences in dissolution data between equipment from different manufacturers. The panel agreed that this was indeed an issue that posed the question, "where do we draw the line?" Terry Way stated that various approaches have been instituted to minimize such variations by requiring certain standardization procedures relating to equipment use such as installation qualification (IQ), operational qualification (OQ), and performance qualification (PQ) at the time of equipment purchase and installation. Although these had become common procedures, he stated that the interpretation of these qualification requirements have changed somewhat over the years. He noted that there used to be two other qualification requirements, design qualification (DQ) where you specify the standards required for the equipment before buying and maintenance qualification (MQ), which have fallen away-MQ being bundled up with $\mathrm{PQ}$. The latter relates to "have you got the equipment that does what you bought it to do?", whereas IQ relates to "have we received what we ordered?" and OP, which relates to "does it perform to specification?" PQ has thus been wrapped up with "does it perform to specifications on a continuing basis over time?"-previously MQ.

Hence, for a particular product, one needs to decide on the limits to specify on your particular equipment for your particular purposes and never mind the compendial specifications. The compendial specifications are intentionally broad to encompass the range of equipment available on the market. Importantly, specifications for a particular product on your equipment need to be set such that it gives confidence that when results are compared, you are comparing "like with like" based on the product you are testing and not because there are possible differences in equipment that you are using to test that product.

Vivian Gray commented that in her experience, she had seen differences between dissolution baths where different baths give different results with a product yet pass the calibrator tests. There have been initiatives to determine if there is a way to make baths more consistent. Similarly, differences in results with different vessels have 
been seen. USP is trying to identify the worst offenders (e.g., vessel diameter) or whatever it is that causes so much variability.

A question was raised as to how to deal with BCS 4 drugs relative to the type of dissolution media to use, such as biorelevant media or surfactants.

Dr.Krämer responded that surfactants were initially not permitted, but public opinion changed and now there are companies that use surfactants in their specifications where the amounts and conditions are justified.

\section{SESSION 3}

The first speaker of this session was Professor C.M. Dangor, Chairperson of the South Africa Medicines Control Council's (MCC) Pharmaceutical \& Analytical Committee whose talk was entitled: THE ROLE OF DISSOLUTION TESTING: AN MCC PERSPECTIVE.

Professor Dangor outlined the aims of the MCC Dissolution Guideline where the intention is to share the current thinking of the regulator, emphasizing that the guideline was not intended as an exclusive approach. Additional information in keeping with knowledge current at the time of an application may be requested, and the use of alternative approaches may be acceptable if scientifically justified. Importantly, the guideline is also intended to provide guidance when in vitro data may be used instead of in vivo. He further alluded to the use of dissolution studies as a research tool to elucidate the mechanism of drug release and its utility in the selection of a model and establishment of specifications.

In special cases, such as for a poorly soluble API, multipoint profiles may be required or consideration given to the use of USP Apparatus 4 (flow-through cell) for dissolution method development.

Professor Dangor described the MCC requirements. Dissolution data are used to demonstrate similarity between reference products from countries to which the MCC aligns itself and to collect information on batch-to-batch consistency of the "test" (generic) and "reference" (brand) products. Dissolution data are also used to waiver proportionally similar dosage forms under the following conditions: APIs and excipients are in exactly the same proportion between products being compared. If not, then the ratios of excipients to total mass of dosage form must be within limits defined under the "Major and Minor Changes Guideline." For products containing highly potent APIs, the masses of different dosage forms should be similar, although API amounts can differ. Professor Dangor provided a table of examples showing the permissible differences in components of a formulation.

A waiver based on dissolution data for a lower strength of a generic product is acceptable to the MCC provided a bioequivalence study was successfully carried out on the higher strength of the dosage form. When a dosage form contains what is considered a highly toxic API, in vitro data based on higher strengths would be considered. In general, the MCC also accepts a biostudy performed on a lower strength when clinical safety or efficacy data including justification of dose desirability of the higher strength are supplied and the API follows linear kinetics over the drug therapeutic range. In the case of modifiedrelease (extended-release) products, capsules containing beads can also be waived based on a single-dose, fasting BE study on the higher strength provided the other dosage strengths are proportionally similar.

Professor Dangor described the situation when a foreign reference product could be used based on dissolution data that shows similarity or equivalence between the foreign reference product and the brand product registered in South Africa. The provisos for acceptance of a foreign reference product include the following considerations:

The foreign reference product must originate from a country whose registration authority is acceptable to the MCC, such as the SADC, FDA, MHRA, MPA, TGA, Canada, EMEA, and Japan. Documents on licensing arrangements (same innovator/API/product; same company/corporate entity, etc.) with SA counterpart must be submitted as well as copies of Labels, PI and PIL, and CoAs for both RPs (SA and foreign) OR a product on the WHO Comparator List is also acceptable for use as a reference product OR if no innovator is identified in the context of above, the choice must be carefully made and comprehensively justified.

When there are post-registration amendments, dissolution data are used to approve such changes. When changes in formulation, site, and method of manufacture are made, the impact on quality can be provided by submitting dissolution data as per original submission, single-point study data. For quality and performance impact, multipoint dissolution profiles in media as per original submission must be provided. For situations where change(s) may affect quality, formulation, and performance, multipoint dissolution in three media is required, and in some instances, bioavailability/bioequivalence studies may be required unless otherwise justified. Biowaivers are possible if in vitro-in vivo correlations have been established. In all cases, comparative data in all three media showing the original versus amended product (viz. pre-change versus post-change product), must be provided.

Professor Rod Walker (Rhodes University) was the next speaker and provided a CRITICAL APPRAISAL OF DISSOLUTION TESTING REQUIREMENTS IN SOUTH AFRICA.

He began with a historical account of drug registration requirements in South Africa. Whereas clinical data were required for the registration of a new chemical entity (NCE), proof of safety and efficacy of generic drug products was based on requirements listed in circulars issued by the Medicines Control Council (MCC). In particular, reference was made to Circular 14/95 where 
dissolution data were accepted as proof of efficacy provided that a monograph for the active in the USP included a dissolution requirement, and that the active was not on a list attached to the circular. Dissolution was to be carried out in three media, one of which had to be that specified in the USP monograph, whereas two other media could span a wide $\mathrm{pH}$ range, such as an acidic medium (e.g., gastric fluid USP), water, or an alkaline medium (e.g., intestinal fluid USP), but cannot be the same as specified in the monograph. The results were to be tabulated and presented in graphical form and include the individual results, the mean, and the standard deviation of the dosage forms. The mean data showing the reference and test products were to be presented in separate graphs for each medium. If the active was insoluble in the other two media, a motivation could be submitted to the MCC for the omission of further testing in the other two media. Importantly, there were no specified criteria for acceptance ( $10 \%$ difference often used).

Professor Walker described the current situation where various guidelines have been established by the MCC but emphasized that there is no single MCC guideline that describes dissolution per se. He noted that dissolution is probably the most important in vitro test that can be used to assess and control variables associated with formulation excipients, design, and manufacturing, which may alter the release characteristics of an active pharmaceutical ingredient from a dosage form and therefore impact on in vivo performance. Professor Walker then addressed several current issues and concerns relating to the MCC dissolution requirements. In particular, he addressed the issue of the use of a "non-South African" reference product in bioequivalence studies where the following options and order of preference are stated in the guideline:

1) Use of the innovator product registered and procured in SA as the reference product.

2) Use of a reference product registered in S.A. but procured in a country with which the MCC aligns itself.

3) Use of a reference product from the WHO comparator list.

4) Use of any other reference product when motivated and justified.

He showed examples of innovator products marketed in different countries where the respective dissolution profiles were significantly different under the same testing conditions. One important concern was that the dissolution requirement to show equivalence between a foreign reference product and the South Africa innovator product permitted dissolution data to be used irrespective of the properties of the API, for example, solubility. This was of concern for interchangeability if no single standard is used and is compounded further by the use of pharmaceutical alternatives. Further concerns relate to the current use of dissolution for postregistration amendments where no differentiation has been made between immediate- release (IR) and modified-release (MR) products. Whereas there were similarities to the U.S. FDA SUPAC guidance with respect to minor changes where most changes were unlikely to impact quality and performance, for some changes that are considered major and likely to impact on product quality and performance, dissolution data only were acceptable in either one medium (compendial/ application) or in three media (BCS Class 1 and 3 drugs). Professor Walker concluded by saying that although the current guidelines have improved the situation, there was room for improvement, as some gaps still existed. He emphasized that international experience should be used to guide decisions and recommended that the impact of the application of the current guidelines be assessed. His final remarks were that the registration of medicines must be based on sound science and current thinking. He strongly recommended that the current guidelines be revisited, and in particular, a designated reference product for use in BE studies, rather than using foreign reference products, was long overdue.

\section{SESSION 4}

The final presentation of the first day, entitled THE ROLE OF DISSOLUTION TESTING: A USP PERSPECTIVE, was

delivered by Dr. Roger Williams (CEO, U.S. Pharmacopeia).

Although Dr. Williams was not able to travel to South

Africa, his presentation was delivered live over the internet and included an interactive question and answer session at the end of his talk. This was facilitated using SKYPE and included audio and visual transmission of both his presentation and the interactive session with participants.

Dr. Williams provided a historical perspective and overview of the establishment of a Drug Bioequivalence Panel in 1974. He referred to the WHO Interchangeability Document where 60-70\% (130 molecules) on the WHO Essential Medicines List was considered suitable for BCS Biowaivers. This, he contended, opens the door for national and global systems of interchangeable pharmaceutical products for nonsolution, orally administered dosage forms. Important assumptions were that there was no subject by formulation interaction (excipients). However, provisions for all other dosage forms were not included. Dr. Williams then considered metrology, which involves the science of measurement that embraces both experimental and theoretical determinations at any level of uncertainty in any field of science and technology.

Turning to performance verification testing relating to dissolution, Dr. Williams described characterization and specifications as being two parts of an overall product control strategy, and that for drug products, BA and BE are one-time product-performance (characterization) studies. He contended that the drug product specification is a set of tests, procedures, and acceptance criteria that confirms the quality of the drug product, and that in the drug product specification, dissolution is a key test to assess drug release (e.g., USP General Chapter <711>). It helps 
assure continuing equivalence relative to clinical trial material. He emphasized that ICH 4B Annex 7 relies solely on mechanical calibration, as does the FDA guidance. The Advisory Panel to the USP Biopharmaceutics Expert Committee considers the approach similar to proficiency testing. Using the ISO framework, Dr. Williams referred to the ISO Guide 43-1, Proficiency Testing by InterLaboratory Comparisons, where proficiency testing is described as the use of interlaboratory comparisons "to determine the performance of individual laboratories for specific tests or measurements and to monitor laboratories' continuing performance."This document indicates,"Participation in proficiency testing schemes provides laboratories with an objective means of assessing and demonstrating the reliability of the data they are producing." Furthermore, it states, "One of the main uses of proficiency testing schemes is to assess laboratories' ability to perform tests competently... It thus supplements laboratories' own internal quality control procedures by providing an additional external measure of their testing capability." As a consequence, the USP Apparatus Suitability Test has been renamed as Performance Verification Test (PVT), the USP reference materials are not calibrators, and as per ISO, the USP Performance Verification Test is also a proficiency test.

The following changes and conclusions were then described by Dr.Williams:

- Salicylic acid RS tablets have been removed as a requirement from $<711\rangle$, official December 1, 2009.

- The chapter now explicitly relies on the technical data sheet of each RS for the details regarding the PVT requirements.

- USP is planning to use a geometric mean and standard deviation acceptance criterion when new lots are released.

- Calculation information will be on the technical data sheet accompanying the standards.

- The PVT calculation toolkit will be also available online to be used to evaluate the PVT test results.

Dr. Williams then provided a brief description of the PVT using USP Prednisone Tablets.

His final comments were that all dosage forms are special and that drug release testing is increasingly important for all dosage forms. The USP will supply a performance test for all dosage forms by route of administration (e.g., USP $<711>$ ), and this performance test will be allied with a PVT with reference material(s), formerly calibrators (e.g., USP web toolkit). He emphasized that reference material is key to allow comparisons across procedures since mechanical calibration does not allow this. Mechanical calibration does not signal problems, and an in-house reference material (FIP) only allows in-house comparisons.

\section{Panel Discussion}

Discussions were mainly on developing acceptable and reproducible dissolution testing methods. Dr. Williams primarily addressed issues mainly on the problems currently with batches of standard tablets for validation. Professor Kanfer had a few remarks on the role of dissolution testing in issues related to Biowaivers and SUPAC.

\section{DAY 2}

\section{USE OF DISSOLUTION TESTING IN COMPLEMENTARY (CAMS) AND TRADITIONAL MEDICINES (TMS).}

\section{SESSION 5}

The first speaker was Dr. Gordon Amidon (University of Michigan) who discussed the APPLICATION OF THE BCS TO SUPPORT THE SAFETY AND EFFICACY OF ALTERNATE MEDICINES (CAMS, NUTRACEUTICALS, ETC.).

Dr. Amidon began by posing the question,"How do we ensure the quality of traditional medicines?" He explained the role of bioavailability/bioequivalence in this context and contended that historically, a Relative Bioavailability (BA)-Based View "misses the underlying scientific issues of dissolution in vivo." Since BE testing involves the "same" drug, once absorbed, its pharmacokinetics is the same. He emphasized that the science of BE is at the absorption site, which for an oral dosage form, is in the Gl tract, and it is necessary to find a best BE test.

Dr. Amidon referred to the August 2000 FDA guidance on a waiver of in vivo BE studies for immediate-release solid oral dosage forms based on the BCS. He then compared that guidance with the EMEA/CPMP note for guidance on the investigation of $\mathrm{BA} / \mathrm{BE}$ and also with the WHO Expert Committee on Specifications for Pharmaceutical Preparations 40th Report, specifically, Annex 7 page 347 and Annex 8 page 391, where BE recommendations based on dissolution testing can be found. In that document, solubility and permeability considerations are the same as FDA and EMEA where dissolution and solubility is studied at pH 6.8 (rather than 7.5). Dissolution is described as either Very Rapid, Rapid, or Not Rapid. The report also recommends classes for drugs on the WHO Essential Medicines List (EML) as well as dissolution 'Biowaivers' for Class 1, 3, and some Class 2 drugs (Ila) and extends BCS Biowaivers to $60 \%$ of those drug products on the EML.

Dr. Amidon then discussed the implications of the BCS for World Drugs where he referred to a Drug Database containing molecular properties and BCS classification of oral immediate-release (IR) drugs in 200 top-selling US, GB, $E S, J P$, and KR drug products. He referred to the use of the Biopharmaceutics Drug Disposition Classification System (BDDCS) in classifying the permeability of marketed drugs (Benet et al., 2008 Pharm. Res. 52 (3)) and compared results from the BCS to classify 165 drugs according to the various classes. Dr. Amidon showed calculations of partition coefficients based on various methodologies and correlation plots of in silico versus measured Log $P$ using 
calculated partition coefficients on 153 drugs. He then presented a graph showing the comparison of the provisional BCS classification of in silico versus referenced solubility approaches on 185 oral IR drugs.

The application of the in silico approach to Traditional Medicines was then described where a traditional Chinese medicine (TCM), Danshen, was used as an example. Dr. Amidon explained that the pharmacokinetic information on Danshen is insufficient and inconsistent, and the absorption mechanism has not been clearly illustrated. In addition, there are quality control issues and problems (e.g., which chemical marker should be a more relevant marker to the in vivo performance of Danshen). There were several questions relating to bioavailability of TCMs that needed to be answered, such as (1) what are the active components responsible for the effects of a TCM product, (2) which active components can be absorbed and display pharmacological activity in vivo following oral administration, and (3) are there any differences in the bioavailability of the active components when given in extract mixture form or in pure form? Dr. Amidon concluded that a dissolution specification can ensure pharmaceutical quality and even possibly safety, whereas an indication of efficacy was questionable and listed as "maybe" and that "the dissolution test is the way forward" for such compounds.

The next speaker was Professor Izzy Kanfer (Rhodes University and Complementary Medicines Committee of the MCC), and his presentation was entitled THE ROLE OF DISSOLUTION TESTING IN COMPLEMENTARY AND AFRICAN TRADITIONAL MEDICINES: DEVELOPMENT OF METHODS AND APPLICATIONS.

Professor Kanfer began his presentation by asking whether dissolution is simply a tool for quality control or could be used as a bioequivalence predictor. He stated that, whereas, in general, the dissolution test is used to measure the release of an active substance (usually single ingredient) from the product in solid oral, tablet, or capsule dosage forms, in his opinion, it is one of the most important and useful in vitro tests, specifically for assuring product quality.

Professor Kanfer indicated that in vitro dissolution is also a valuable tool to assess drug product stability and shelf life. As products age, formulation properties may change and alter the dissolution characteristics of the product over time. For example, moisture levels may increase or decrease, and this can result in altered tablet hardness and possible changes in drug release rates. In addition, polymorphic transformations can affect solubility and dissolution, and such changes can usually be monitored using dissolution testing. He stated that there were various factors that affect dissolution rate. Over the past 30 years or so, dissolution testing has confirmed its utility to assess and control variables associated with formulation excipients, design, and manufacturing, which may alter the release characteristics of the active moiety from the dosage form. Citing magnesium stearate as an example, Professor Kanfer illustrated the effect of lubricant concentrations on dissolution rates and the subsequent correlation of such effects on drug absorption. He also showed how different types of starches could affect dissolution rates with resultant bioavailability implications for the manufactured product.

Professor Kanfer referred to the FDA Guidance for Industry, Dissolution Testing of Immediate-Release Solid Oral Dosage Forms (U.S. Dept of Health and Human Services, Food and Drug Administration, U.S. Government Printing Office: Washington, DC, 1997), which recommends the use of buffers in the $\mathrm{pH}$ range 1.2-7.5 for dissolution media and initial method development. He emphasized that unlike orthodox medicines, specific guidelines for dissolution testing of complementary/alternate (CAMs) and traditional medicines (TMs) have not been developed. Furthermore, he continued, in most cases, there are no dissolution testing requirements for the quality control of such products. He recommended that there is an urgent need to consider the application of dissolution testing as a quality control tool in CAMs and TMs. However, he cautioned that unlike conventional pharmaceutical products that usually consist of one or two well-characterized active ingredients, CAMs/TMs are much more complex and usually consist of multiple components that may be active individually or in combination. Hence, the question "What should be measured?" needs to be addressed for such medicines. In contrast to chemically defined components, the "actives" in CAMs/TMs are generally defined to be the whole herbal preparation (e.g., the extract in its entirety).

Professor Kanfer used St. John's Wort (SJW) products as an example and described the chemical composition of this plant, which contains, among other things, several identified phytochemical components, such as phloroglucinols, naphthodianthrones, and flavonoids, where the various components have been related to pharmacological or synergistic activity. This particular example clearly shows how quality control procedures can and should be developed for such products. Professor Kanfer pointed out that the various properties, both pharmacologic and physicochemical (e.g., polarity, solubility), need to be considered in developing QC markers and tools (e.g., naphthodianthrones are medium polarity whereas the flavonoids are generally hydrophilic, etc.).

He stated that whereas content uniformity and compliance with label claim are standard quality criteria that need to be tested, dissolution testing should also be used.

Professor Kanfer described how dissolution testing was successfully applied for the QC of African Potato (AP), a commonly used African traditional medicine (ATM). Importantly, he showed how it is possible to use different compositions of dissolution media when the components of the dosage form have different polarities or solubilities 
where the usual aqueous compendial media could not be used for all the components.

Hence, dissolution media such as FaSSIF (fasted-state simulation, generally pH 6.5) and FeSSIF (fed-state simulation, generally $\mathrm{pH}$ 5.0) were chosen and successfully used for the investigation of the dissolution of the relevant components, hypoxoside and $\beta$-sitosterol, present in African Potato. He also presented data on the dissolution testing of Sceletium products, another important ATM used in South Africa. In this instance, although Sceletium species contain various mesembrine alkaloids, the in vitro release of mesembrine itself was successfully used as a QC marker for products containing Sceletium plant extracts.

Professor Kanfer then summarized by stating that depending on a particular product and its components, suitable dissolution methods can be developed as valuable QC tools for such complicated products. He added that even when dealing with complex products such as CAMs and ATMs, it is clear that dissolution testing has an important role as a QC tool, but it is essential to decide what to monitor and also to consider whether any biorelevance could be associated with the resulting data.

\section{ROLE OF PERFORMANCE TESTING IN RESEARCH AND DEVELOPMENT.}

\section{SESSION 6}

Dr. Nikoletta Fotaki (University of Bath, UK) was the first speaker in this session and her talk was entitled

\section{FLOW-THROUGH CELL APPARATUS: APPLICATIONS FOR IVIVCs/IVIVRs.}

Dr. Fotaki outlined the various roles of dissolution testing from its use in $\mathrm{QC}$, as a guide in formulation development, and its assistance in establishing IVIVCs for biowaivers. Dr. Fotaki then addressed the flow-through cell apparatus (USP Apparatus 4). She described various aspects such as the introduction of a flowing medium apparatus by the FDA in 1957, where sink conditions were maintained, and stressed that the dissolution rate reflects the behavior of the sample and not the solubility of the substance. Furthermore, the flow-through cell apparatus could operate in a controlled hydrodynamic environment. Dr.Fotaki pointed out that in particular, the flow-through cell apparatus is very useful for low solubility drugs, microparticulates, implants, suppositories, and controlled-release formulations. She then described the key features of the apparatus, such as the use of an open system for samples that require a high volume of medium and a closed system for samples requiring a low volume of medium (i.e., $27 \mathrm{~mL} / \mathrm{sample)}$. The key difference between these configurations is that with an open system, raw data are obtained in noncumulative form (amounts dissolved at specific time intervals) compared with the usual cumulative profiles. Dr.Fotaki explained that the flow-through cell could also be operated under laminar flow conditions where the sample is situated on a bed of glass beads in the cell column or simply placed in the open column under turbulent flow conditions. She discussed several advantages such as (1) the ability to change media or flow rate within a single run, (2) ability to efficiently simulate intralumenal hydrodynamics, (3) use of long duration test runs without the problem of evaporation, (4) provision for special dosage forms (floating dosage forms, implants, suppositories), and (5) avoidance of carry-over effects facilitating IVIVC — a single profile corresponds to the release in the entire Gl lumen. However, there are several disadvantages such as the high volume of media required when used as an open system and the need for more training compared to using the standard USP Apparatus 1 or 2. Using the flow-through cell apparatus was labor-intensive, and filtration efficiency may be an issue when using USP 4 with biorelevant media.

Dr.Fotaki then presented several case studies illustrating the use of Apparatus 4.

The next presentation entitled THE ROLE OF INTRINSIC DISSOLUTION RATE IN THE ASSESSMENT OF SOLID ORAL DOSAGE FORMS was delivered by Dr. Mike Skinner of the Biopharmaceutics Research Institute at Rhodes University. He began by defining intrinsic dissolution (ID) rate, which is rate of dissolution of pure drug substance from a constant surface area. He explained that ID requires the drug to be compressed into a disc to provide a single exposed surface, and ID is normally expressed as $\mathrm{mg} / \mathrm{cm}^{2} /$ min, also termed "Flux." He indicated that the use and application of ID is increasing, especially with the more highly insoluble drugs such as those in BCS Class 2, which have low solubility (LS) and high permeability (HP), where these types of drugs are associated with absorption problems. He stressed that ID is an important tool for raw material characterization and that there are now some compendial methods (USP, BP,EP) that include ID testing. He further informed the audience that the USP 32 General Chapter $<1087>$ contains information for Apparent Intrinsic Dissolution-Dissolution Testing Procedures for Rotating Disc and Stationary Disk using USP Apparatus 4. In addition, ID is described in the British Pharmacopoeia Appendix XII B (5) and in the European Pharmacopoeia, Method 2.9.29.

Dr. Skinner addressed the issue of intrinsic dissolution rate (IDR) and bioavailability. He referred to a 1972 article by Kaplan who proposed limits for dissolution rate-limited absorption using IDR measurements with centrically mounted discs at a speed of $50 \mathrm{rpm}$. Bioavailability was likely to be reduced when the IDR was $<1.0 \mathrm{mg} / \mathrm{cm}^{2} / \mathrm{min}$ and highly likely to be reduced when the ID rate was $<0.1$ $\mathrm{mg} / \mathrm{cm}^{2} / \mathrm{min}$ throughout the $\mathrm{Gl} \mathrm{pH}$ range of 1.2-7.4.

Dr. Skinner concluded that the determination of IDR is very valuable for use in formulation development since it can easily assess the influence of excipients using discs of drug/excipient composites, which can be readily prepared. He indicated that the use of ID in API raw material evaluation is increasing with the proliferation of raw material 
producers and that ID rate determination offers a simple test to characterize an important drug property.

Furthermore, he suggested that it can be used to assess numerous formulations relatively easily before preparing experimental/biobatches.

\section{SESSION 7}

The following speaker was Dr. Johannes Krämer (PHAST, Germany) whose presentation was entitled:

ESTABLISHING A RELATIONSHIP BETWEEN DISINTEGRATION AND DISSOLUTION.

Dr. Krämer stated that when considering establishing a relationship between disintegration and dissolution, a risk evaluation is a prerequisite. This should include the vital indication for the drug, its status regarding therapeutic index (e.g., narrow), stability in the Gl tract, existence of an absorption window, and evaluation of the dosage form and excipients. He discussed the various goals where the intention may be to predict changes in bioavailability or the evaluation of robustness as a parameter of drug product-related safety. Further goals may include its use to investigate critical manufacturing variables such as proving uniformity of product quality within the technological range of manufacturing processes for QC purposes. One very interesting goal is the possibility of predicting "Bioperformance" based on the BCS. Dr. Krämer discussed the BCS and related dissolution requirements and described how it serves as a base to establish specifications for in vitro dissolution testing. He referred to the ICH Q 6A Guideline in which a Decision Tree (\# 7) is described for setting acceptance criteria for drug product dissolution and described how following the decision tree could lead to general disintegration acceptance criteria with an upper time limit being acceptable. However, Dr. Krämer emphasized that this should not be considered as a correlation per se, since disintegration in QC is defined as time of completeness of a kinetic process, and dissolution in QC is the amount of completeness at a specified time, hence there are no analogous parameters available for a correlation. The key question that needs to be answered is whether a relationship between disintegration and dissolution has been established. He showed how, theoretically, the dissolution process of a solid oral dosage form should result in a sigmoid curve of percent drug dissolved versus time. He then discussed a case study involving a BCS Class 1 drug. The aim of that study was to prove that disintegration is the prerequisite for dissolution. This required defining comparable parameters (i.e., amount dissolved at the time of complete disintegration in the dissolution apparatus) and comparing analogous parameters for freshly manufactured batches, samples after accelerated stability treatment, and historical data of batches successfully released. The proof of assumptions is based on the amount dissolved at the time of complete disintegration in the disintegration apparatus and is comparable to the process analyzed for the dissolution apparatus.

Dr. Krämer explained the use of statistical moments and the "Additivity of Mean Times." In the example, the mean disintegration times showed higher variability than the mean dissolution times at $\mathrm{pH}$ values of 1.2 and 6.8, implying that the disintegration method is more variable. However, from the mean disintegration time data in the dissolution apparatus at $\mathrm{pH} 4.5$ and 6.8, Dr. Krämer explained that the disintegration process is the most variable subprocess, whereas the mean terminal dissolution times after disintegration in the dissolution apparatus at $\mathrm{pH} 45$ and 6.8 are much less variable. He thus concluded that disintegration is the prerequisite for dissolution and that at the time of complete disintegration, a rather constant amount of drug is being dissolved. This, he said, is valid for the dissolution apparatus and for the disintegration apparatus. The subprocesses, however, overlap, but with the help of statistical moment theory, the sub-processes can be clearly described. However, disintegration is the most variable subprocess, and the relationship between disintegration and dissolution is cause-and-effect.

Hence, Dr. Krämer asserted that, as a consequence, the dissolution test may be waived for batch release testing for all formulations included in the study. He suggested that for setting specifications, dissolution testing as a biopharmaceutically relevant test procedure may be waived since disintegration is a prerequisite for dissolution and recommended for batch release testing. Furthermore, comparison of the disintegration results together with the dissolution results for the disintegration apparatus allows tightening of specifications to $5 \mathrm{~min}$ (Ph. Eur./USP: $15 \mathrm{~min}$ ). Thus for all immediate-release dosage strengths of the particular API-containing product discussed, the specification for disintegration time was set as not more than (NMT) 5 min.

The final presentation, IN VITRO SIMILARITY AS SURROGATE FOR THERAPEUTIC EQUIVALENCE: An Investigation of the South American Markets was provided by Dr. Raimar Löbenberg of the University of Alberta, Canada. As in the case of Dr. Williams on the previous day, Dr. Löbenberg was not able to travel to South Africa, but he delivered his presentation "live" over the internet and included an interactive question and answer session at the end of his talk. This was facilitated using SKYPE and included both audio and visual transmission of his presentation followed by an interactive session with participants.

Dr. Löbenberg explained that the study was designed to investigate in vitro dissolution differences between BCS Class 1 drugs on the South American Market. The products were compared to U.S. products and involved three drugs, zidovudine, amoxicillin, and metronidazole. The U.S. 
reference listed drug (RLD) was identified using the Orange Book and where necessary, an alternative comparator product was chosen. The main objective of this study was to investigate the dissolution of the aforementioned drug products.

Dr. Löbenberg presented dissolution data on zidovudine capsules performed in three different media, simulated gastric fluid (SGF), pH 4.5 buffer, and simulated intestinal fluid (SIF). He compared SGF with pH 1.2 buffer and SIF with pH 6.8 buffer, explaining that SGF also had a $\mathrm{pH}$ of approximately 1.2 but contained sodium chloride and pepsin, whereas SIF pH was prepared in accordance with the buffer of $\mathrm{pH} 6.8$.

Dissolution testing was carried out using USP Apparatus 2 at $75 \mathrm{rpm}$, and samples were collected at 10, 15, 20, 30, 45 , and 60 min. Retrovir (GSK -USA) was used as the RLD. Dr. Löbenberg presented a slide showing manufacturing differences between the zidovudine products (capsule sizes and shells as well as the blister packaging) and also visible discolorations and other deficiencies. Interestingly, macroscopic differences were even apparent between the GSK USA and GSK England products. However, all products, when compared to the RLD (GSK USA Retrovir), passed the WHO criteria for in vitro bioequivalence.

The next set of results discussed was the dissolution tests on amoxicillin tablets. Dissolution was carried out using USP Apparatus 2 at $75 \mathrm{rpm}$ in SGF, in pH 4.5 buffer, and in SGF. Ten generic products failed to meet the in vitro similarity requirements in accordance with the WHO guidelines.

Metronidazole tablets USP was the third group of products tested using the same methods and conditions used for the zidovudine and amoxicillin products. The RLDs were Flagyl $250 \mathrm{mg}$ (Searle USA) and $500 \mathrm{mg}$ (Searle, Pharmacia, and Pfizer USA). In summary, none of the metronidazole products were equivalent to the respective RLDs; 12 products failed the in vitro similarity requirements. Furthermore, two strengths of the RLD products (i.e., the lower and higher strengths) did not meet the in vitro similarity requirements in two of the media.

Dr. Löbenberg's results clearly showed that many BCS 1 generic drug products are not similar in vitro to the USA RLD or alternates. He concluded that dissolution testing and pharmaceutical equivalence criteria, together with in vitro similarity, could be suitable surrogates to ensure bioequivalence.

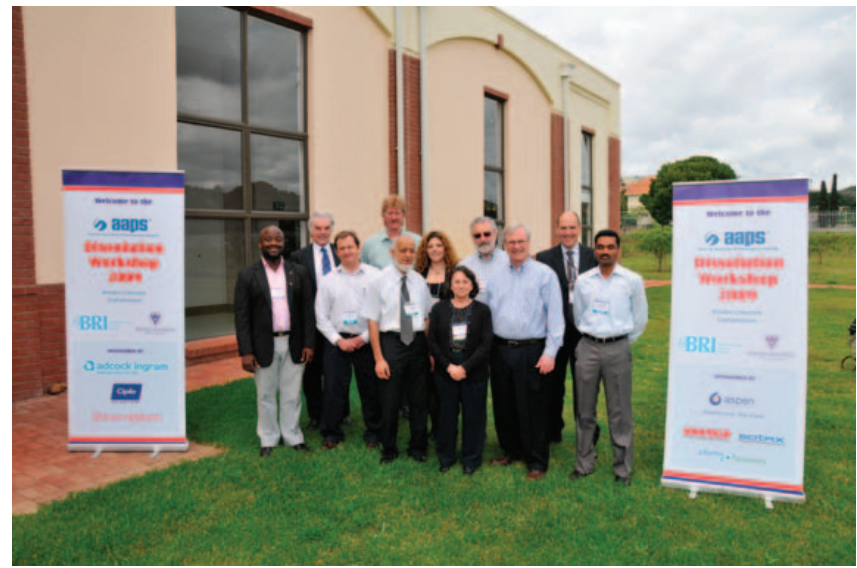

Workshop speakers and organizers, from left to right: Ralph Tettey-Amlalo, Terry Way, Mike Skinner, Rod Walker, Cass Dangor, Nikoletta Fotaki, Vivian Gray, Izzy Kanfer, Gordon Amidon, Johannes Kramer, Srinivas Patnala.

\section{PANEL DISCUSSION}

The discussion revolved mainly on differences and similarities of dissolution profiles of generic products manufactured at different locations (countries).

The questions were related to consolidation of such data to study the impact of active and inactive pharmaceutical ingredients. There was also a major concern on how such results would affect the local (South African) regulations and MCC approvals for such variable products.

Vivian Gray gave concluding remarks thanking the speakers, especially those who traveled a long distance, and the audience members for their active participation.

Additional Sponsors of this workshop were:

Biopharmaceutics Research Institute, Rhodes University, Grahamstown, South Africa

Rhodes University, Grahamstown, South Africa

Adcock Ingram Limited, Johannesburg, South Africa

Aspen Pharmacare, South Africa

Anatech Analytical Technologies, Johannesburg, South Africa

Pharma Dynamics, Cape Town, South Africa

Cipla Medpro, Cape Town, South Africa

Johnson \& Johnson, Cape Town, South Africa

Sotax Solutions for Pharmaceutical Testing, United Kingdom

The slides of this workshop can be found on the AAPS In Vitro Release and Dissolution Testing Focus Group website. www.aapspharmaceutica.com/inside/focus_groups/ InVitro/index.asp 